\title{
Potential targeting of FLT3 acute myeloid leukemia
}

\author{
Alexander J. Ambinder and Mark Levis \\ Department of Oncology, Sidney Kimmel Comprehensive Cancer Center, Johns Hopkins \\ University School of Medicine, Baltimore, MD, USA
}

\section{ABSTRACT}

\begin{abstract}
A berrant FLT3 receptor signaling is common in acute myeloid leukemia (AML) and has important implications for the biology and clinical management of the disease. Patients with FLT3-mutated AML frequently present with critical illness, are more likely to relapse after treatment, and have worse clinical outcomes than their FLT3 wildtype counterparts. The clinical management of FLT3-mutated AML has been transformed by the development of FLT3 inhibitors, which are now in use in the frontline and relapsed/refractory settings. However, many questions regarding the optimal approach to the treatment of these patients remain. In this paper, we will review the rationale for targeting the FLT3 receptor in AML, the impact of FLT3 mutation on patient prognosis, the current standard of care approaches to FLT3-mutated AML management, and the diverse array of FLT3 inhibitors in use and under investigation. We will also explore new opportunities and strategies for targeting the FLT3 receptor. These include targeting the receptor in patients with non-canonical FLT3 mutations or wild-type FLT3, pairing FLT3 inhibitors with other novel therapies, using minimal residual disease testing to guide the targeting of FLT3, and novel immunotherapeutic approaches.
\end{abstract}

\section{Introduction}

FLT3 (FMS-Like Tyrosine kinase-3) is a type 3 receptor tyrosine kinase that plays an important role in the expansion of multi-potent progenitor cells within the bone marrow (BM) ${ }^{1,2}$ It is also among the most commonly mutated genes in acute myeloid leukemia (AML). ${ }^{3}$ In an AML patient with a FLT3-activating mutation, the clinician is immediately faced with a variety of challenges and questions. These patients routinely present critically ill with hyperleukocytosis. Management decisions must be made rapidly, and trial participation is often difficult to arrange. There is agreement that FLT3 inhibitors need to be incorporated into treatment, but how should that be done if a patient is receiving a lower-intensity regimen such as a hypomethylating agent (HMA) with or without venetoclax? What impact does the FLT3 mutant allelic burden have on treatment decisions? What about co-mutations like NPM1 or DNMT3A? Should the patient receive an allogeneic transplant? A FLT3-activating mutation might be present at diagnosis and absent at relapse, or vice versa. A FLT3 mutation can impair the responses to other targeted agents like IDH inhibitors or venetoclax. Nothing seems to complicate the management of an AML patient more than the presence of a FLT3 mutation. This receptor's activity, both mutated and non-mutated, is intertwined throughout this disease, and the potential benefits of targeting it in more refined ways is just beginning to be realized.

Why FLT3 is important to target in acute myeloid leukemia

FLT3 receptor's natural ligand is FLT3 ligand (FL). Binding of the ligand leads to homodimerization of the receptor, autophosphorylation, and the transduction of pro-survival and proliferative signals via the RAS/MAPK, JAK/STAT5 and PI3K/AKT pathways. ${ }^{4,5}$ In healthy individuals, FLT3 is expressed by a limited subset of hematopoietic stem cells (HSC), multipotent progenitors (MPP), common lymphoid progenitors (CLP), common myeloid progenitors (CMP), and mature dendrit-
Haematologica 2021

Volume 106(3):671-681

\section{Correspondence:}

ALEXANDER J AMBINDER

aambind1@jhmi.edu

\section{MARK LEVIS}

mlevisma@jhmi.edu

Received: May 4, 2020.

Accepted: July 7, 2020.

Pre-published: July 23, 2020.

https://doi.org/10.3324/haematol.2019.240754

(C)2021 Ferrata Storti Foundation

Material published in Haematologica is covered by copyright. All rights are reserved to the Ferrata Storti Foundation. Use of published material is allowed under the following terms and conditions:

https://creativecommons.org/licenses/by-nc/4.0/legalcode. Copies of published material are allowed for personal or internal use. Sharing published material for non-commercial purposes is subject to the following conditions: https://creativecommons.org/licenses/by-nc/4.0/legalcode, sect. 3. Reproducing and sharing published material for commercial purposes is not allowed without permission in writing from the publisher. 
ic cells. ${ }^{6} \mathrm{FL}$ is produced by a much broader array of cell types, including lymphocytes, HSC, and BM stromal cells, and functions as a growth factor that stimulates myelopoiesis. The ubiquity of FLT3 expression on MPP and its role as a stimulant of myelopoiesis identifies this receptor as an obvious candidate driver of leukemogenesis.

Thirty percent of patients with AML harbor mutations in FLT3 (mFLT3) that result in constitutive activation of the receptor and its downstream pathways. ${ }^{7,8}$ The two canonical varieties of mutation are internal tandem duplications (ITD), which typically occur in or near the juxtamembrane domain (JMD), and point mutations in the tyrosine kinase domain 2 (TKD). ITD and TKD mutations occur in about $23 \%$ and $7 \%$ of AML patients, respectively. The potential for FLT3 as a driver of leukemogenesis goes beyond these two mutation types. Non-canonical activating mutations are now being detected through next-generation sequencing (NGS) at diagnosis or are emerging in the setting of FLT3 inhibition. ${ }^{9-11}$ Even in cases in which no FLT3 coding mutation is detectable, the receptor can be overexpressed on the cell surface of leukemic blasts and contributes to the survival and proliferation of the leukemic clone. ${ }^{12-17}$ Furthermore, chemotherapy-induced aplasia stimulates the BM stroma to produce FL, fueling the recovery, selection, and expansion of any AML clone that is positioned to take advantage of it.

The pervasiveness of aberrant FLT3 signaling across the spectrum of AML subtypes defies straightforward classification. FLT3 mutations are most common in AML with normal cytogenetics, but may also occur in the setting of other disease-defining genetic lesions such as inv(16), $t(8 ; 21)$ and $t(15,17)$. They frequently co-occur with other driver mutations such as DNMT3A, NPM1, and IDH1/2. ${ }^{3}$ A FLT3 mutation is almost invariably the final mutation in a series of genetic and epigenetic 'hits', propelling the affected clone from a preleukemic state to full-blown leukemia. While FLT3 mutations may be detected in almost any AML subtype, they are rarely observed in other myeloid neoplasms or clonal states. ${ }^{18}$ FLT3 mutations are uniquely specific to the AML phenotype and their signature is one of hyper-proliferation and worsened outcomes.

Collectively, these features provide ample rationale for targeting the FLT3 receptor. It is also a particularly appealing drug target because it appears to be non-essential. In mice, knock out of FL or FLT3 is non-lethal, but it does reduce the capacity to repopulate an aplastic marrow and results in the marked absence of NK and dendritic cells. ${ }^{6,19,20}$ This thinking has led to the incorporation of FLT3 mutation status into every aspect of AML management and to the investigation of nearly a dozen FLT3 inhibitors in clinical trials across the spectrum of the disease's trajectory. The resulting successes have confirmed the centrality of aberrant FLT3 signaling to the pathogenesis of AML and highlight the importance of addressing unfettered FLT3 signaling throughout the disease's trajectory.

\section{Current standard of care for acute myeloid leukemia and for FLT3-mutated acute myeloid leukemia}

With the exception of acute promyelocytic leukemia (APL), the first branching point in the decision tree for the management of de novo AML is determined by a patient's fitness for intensive chemotherapy and their age. For those who are fit, induction with conventional chemotherapy (most commonly $7+3$ or CPX-351) is standard, whereas those who are elderly or unfit are treated with an HMA or low-dose cytarabine and venetoclax, if the latter is available. At most centers, treating clinicians will not yet have the results of FLT3 mutation testing at the time that this decision is made. So how should a clinician respond when a patient who has already embarked on a therapeutic path is found to have mFLT3?

\section{The fit patient}

Historically, the standard of care for fit patients with mFLT3 AML was conventional chemotherapy alone. Patients with mFLT3-ITD AML respond to induction chemotherapy in a manner similar to their WT counterparts, but their remissions are shorter and their rates of relapse are higher. The discrepancy between remission rates and outcomes is explained by the polyclonal nature of new-onset AML and the relatively low proportion of blasts that harbor mFLT3 at this stage. Chemotherapy induces aplasia and mFLT3 clones eventually expand, possibly driven by increasing expression of FL during successive rounds of aplasia. ${ }^{21}$ The relapsed leukemia that emerges after traditional chemotherapy is oligoclonal and more "addicted" to FLT3 signaling with a larger proportion of blasts harboring mFLT3.

The standard of care for fit patients with mFLT3 AML was re-defined by the phase III RATIFY trial, in which patients with mFLT3-ITD or mFLT3-TKD were randomized to standard induction chemotherapy plus midostaurin or chemotherapy alone. Midostaurin, a first generation FLT3 inhibitor, was administered on days 8 through 21 and was then included in subsequent cycles of consolidative chemotherapy and as maintenance. The addition of midostaurin resulted in higher rates of event-free survival (EFS) (8.2 vs. 3.0 months, $P=0.002)$ and better overall survival (OS) (HR 0.78, 95\% confidence interval [CI], 0.63$0.96)$ compared to chemotherapy alone. ${ }^{22}$ The addition of midostaurin appeared to benefit patients running the gamut of mFLT3 AML including those with ITD and TKD mutations, low or high allelic ratios, and in the presence or absence of other significant co-mutations. ${ }^{22,23}$ Midostaurin was approved in Europe for use in the induction, consolidation and maintenance phases, as administered in the RATIFY trial. In the US, however, it was only approved for use with induction and consolidation, reflecting the US Food and Drug Administration's uncertainty regarding the need for maintenance therapy. This question of whether midostaurin maintenance provides clinical benefit is complicated by the frequency with which these patients undergo allogeneic hematopoietic stem cell transplant (alloHSCT).

\section{The unfit or elderly patient}

For the unfit or elderly patient with de novo AML, venetoclax-based regimens have become the new standard of care. The diminished role of traditional DNA-damaging chemotherapeutic agents as well as venetoclax's novel mechanism of action led to some hope that these regimens might mitigate the negative prognostic significance of mFLT3. This does not seem to be the case. Recent evidence suggests that mFLT3 AML are less responsive to these regimens and that progression on these regimens is frequently driven by the acquisition or expansion of mFLT3 clones. $^{24,25}$ 
Even if a clinician has the results of FLT3 mutation testing prior to initiation of therapy, a superior alternative to a venetoclax-based regimen that makes use of FLT3 inhibitors has not yet been identified. The combination of azacitidine and sorafenib was studied in a phase II trial and has a $2 \mathrm{~A}$ recommendation in the most recent National Comprehensive Cancer Network (NCCN) guidelines, but is unlikely to outperform a venetoclax-based regimen due to sorafenib's limited activity against FLT3..$^{26,27}$ More potent FLT3 inhibitors such as gilteritinib have not been approved for use in the upfront setting and are unlikely to succeed as monotherapy at this early stage due to the relatively low mFLT3 allelic burden. ${ }^{28}$ It is only with the selective pressure of other therapies that the hardier mFLT3 clones come to dominate the tumor bulk. ${ }^{29}$ Currently, the discovery of a FLT3 mutation in an unfit or elderly patient receiving a standard of care venetoclaxbased regimen is a cause for concern, but one for which there is as yet no evidence-based response.

Consolidative strategies: chemotherapy or allogeneic transplant?

The next dilemma that the treating clinician faces in the management of mFLT3 AML is determining the most appropriate consolidative therapy. For the fit patient who received intensive induction with $7+3+$ midostaurin, should they proceed with consolidative high-dose cytarabine and midostaurin or alloHSCT?

This question is one of risk and prognosis. There is general agreement that alloHSCT is most beneficial in patients with AML who are at the highest risk of relapse. FLT3 is a bad actor, but the degree to which it impacts prognosis (and therefore the decision to pursue alloHSCT) may depend upon the specific characteristics of the FLT3 mutation and the company it keeps. In the 2017 European LeukemiaNet (ELN) guidelines' risk classification system, FLT3-ITD allelic ratio (mut/WT threshold of 0.5) is combined with NPM1 mutation status into a single risk variable with three levels. ${ }^{30}$ Patients with a low FLT3-ITD allelic ratio and mutated NPM1 are categorized as favorable, whereas a high allelic ratio and WT NPM1 are categorized as adverse risk. The other two combinations fall within the intermediate category.

This risk classification system was recently validated in a retrospective analysis of the RATIFY trial. ${ }^{23}$ This study found a clear benefit for alloHSCT in patients with adverse risk AML (i.e., high allelic ratio mFLT3-ITD and NPM1 WT) in first complete remission (CR), but no difference in outcomes for favorable and intermediate risk mFLT3 AML. These findings stand in contrast to a substantial body of evidence demonstrating a survival benefit for alloHSCT in patients with mFLT3 without regard for FLT3 characteristics or co-mutations. ${ }^{31-36}$ There are other considerations not included in the guidelines, however, that may account for these discrepant findings.

First, FLT3-ITD have other prognostic characteristics other than allelic ratio. ITD consist of in-frame duplications in exons 14 and 15 and they vary significantly in length. Shorter ITD occupy the JMD $(70 \%)$ alone while longer ITD extend beyond the JMD and encroach upon the TKD. ${ }^{37,38}$ The JMD has an autoinhibitory function that is disabled with the insertion of an ITD leading to constitutive activation. ${ }^{39}$ FLT3-ITD AML with longer ITD length and higher mutant allelic ratio at diagnosis are associated with worse clinical outcomes and may be less responsive to FLT3 inhibitors. ${ }^{28,34,40-43}$
Second, determining the mFLT3 allelic ratio is not straightforward. FLT3-ITD are detected and characterized using polymerase chain reaction (PCR). Shorter ITD are transcribed more efficiently than longer ones, so longer ITD lead to underestimation of the true mutant allelic ratio; this phenomenon is known as PCR bias. ${ }^{44,45}$ Therefore, a patient harboring a long ITD is more likely to have a low mutant allelic ratio and to be classified as lowrisk, when in fact, they may be high-risk and would benefit from an aggressive consolidating strategy. To complicate matters further, mFLT3 is a polyclonal disease and some patients have more than one ITD variant.

Third, the ELN guidelines recognize that the prognosis of FLT3 AML may be modulated by co-mutations such as NPM1, but there are other pertinent mutations that are not incorporated into the guidelines. For example, mutations in FLT3, NPM1 and DNMT3A tend to cluster together more frequently than would be expected by chance alone. ${ }^{46}$ AML bearing mutations in all three genes (so called 'triple-mutant AML') are biologically distinct and have dismal prognoses. ${ }^{3,47,48}$ By ELN guidelines, these patients might be classified as favorable risk, when in fact, they may belong in the adverse-risk category. This phenomenon has recently been recognized and transplant outcomes have not been specifically reported, but following the principle that adverse-risk AML benefits most from alloHSCT, this subset should be considered for alloHSCT.

In our practice, we routinely pursue alloHSCT for patients with mFLT3 AML. Of course, other factors ultimately influence the decision to transplant, such as the patient's medical co-morbidities or difficulties in finding a suitable donor. With a better understanding of the prognostic significance of specific FLT3 characteristics and comutations, a low risk population may be identified that does not need alloHSCT.

\section{Post-transplant maintenance}

Finally, is there a role for targeting FLT3 as maintenance? This is an area of intense interest, but for which equipoise remains. In the phase II SORMAIN study, post-transplant maintenance with sorafenib was compared to placebo and showed an overall survival (OS) benefit ( $\mathrm{HR}=0.447$, $P=0.03$ ), but a major limitation of this study was that patients had not received pre-transplant treatment with a FLT3 inhibitor. ${ }^{49}$ In the era of upfront FLT3 inhibition, this is likely to be a rare circumstance. Another phase II study of post-transplant midostaurin maintenance compared to placebo demonstrated relapse rates of $11 \%$ and $24 \%$, respectively, but the study was not statistically powered to show a difference $(P=0.34)$ and therefore was only able to conclude that maintenance therapy was safe. ${ }^{50}$ In a posthoc analysis, patients with greater inhibition of FLT3 had improved survival outcomes compared to standard of care, suggesting that maintenance with a FLT3 inhibitor may have clinical benefit.

\section{The relapsed/refractory setting}

Relapsed or refractory (R/R) mFLT3 AML is overwhelmingly unresponsive to salvage chemotherapy. In the phase III ADMIRAL trial, patients with R/R mFLT3 AML were randomized to single agent gilteritinib or salvage chemotherapy. The percentages of patients with complete remission and full or partial hematologic recovery were $34 \%$ and $15.3 \%$ (risk difference $18.6 \%, 95 \%$ confidence 
interval 9.8-27.4\%), respectively. Gilteritinib demonstrated improved OS compared to chemotherapy with a median $O S$ of 9.3 months versus 5.3 months $(P<0.001)$ and a hazard ratio for death of 0.65 (95\% CI: $0.39-0.83) .{ }^{51}$ This trial established the importance of targeting mFLT3 and resulted in FDA approval of gilteritinib in the R/R setting. Similarly, in the phase III QUANTUM-R trial, patients randomized to single agent quizartinib had improved OS compared to those randomized to salvage chemotherapy, leading to the drug's approval in Japan. These results are tempered by the fact that all of these patients relapse unless they undergo subsequent transplant. Furthermore, only $12.4 \%$ of patients in the ADMIRAL trial had previously received a FLT3 inhibitor. As FLT3 inhibitors use in the frontline setting increases, the effectiveness of FLT3 inhibitors such as gilteritinib in the $R / R$ setting may diminish.

\section{FLT3 inhibitors}

There are nearly a dozen FLT3 inhibitors in use or in clinical trials. Different pharmacologic properties and adverse effects may be preferred under different circumstances. As more FLT3 inhibitors become available for use in different scenarios, appropriate selection will depend upon a nuanced understanding of their similarities and differences.

\section{First generation FLT3 inhibitors}

The first FLT3 inhibitors were tyrosine kinase inhibitors (TKI) with broad anti-kinase activity that were repurposed for use in mFLT3 AML. These TKI include sunitinib (SU11248), midostaurin (PKC412), lestaurtinib (CEP-701), and sorafenib (BAY43-9006). Midostaurin and lestaurtinib are class I inhibitors that bind to the ATP-binding site in the intracellular active pocket of the enzyme and are active against both ITD and TKD mutations. Sunitinib and sorafenib are class II inhibitors, which bind to the ATPbinding site and interact with an adjacent hydrophobic pocket. This hydrophobic pocket is only exposed in the inactive conformation and is made inaccessible by TKD mutations. Therefore, TKD mutations confer resistance to class II inhibitors. TKD mutations are rarely present alongside FLT3-ITD mutations at diagnosis, but mFLT3-ITD AML may acquire TKD mutations as a resistance mechanism under the selective pressure of class II FLT3 inhibitors. $^{52}$

In vitro, first-generation inhibitors appear to be potent against the mFLT3 receptor. However, in vivo, they are highly protein bound in plasma, reducing their potency. In the plasma inhibitory activity (PIA) assay, $85 \%$ sustained kinase inhibition correlates with clinical response. ${ }^{53,54}$ In plasma, midostaurin and lestaurtinib have $\mathrm{IC}_{\mathrm{s}} \mathrm{s}$ of $1000 \mathrm{nM}$ and $700 \mathrm{nM}$, respectively, ${ }^{53}$ whereas second generation FLT3 inhibitors have $\mathrm{IC}_{\mathrm{sos}}$ in the range of $20-40 \mathrm{nM}$. $^{55,56}$ First generation inhibitors also have shorter half-lives. These characteristics explain their limited clinical efficacy as single agents. ${ }^{57,58}$ Furthermore, their broad anti-kinase activity contributes to worse toxicity profiles compared to those of second generation TKI.

First generation FLT3 inhibitors have been most successful in combination with chemotherapy in the upfront setting. In the phase II SORAML trial, 267 patients with newly diagnosed AML, most of whom had WT-FLT3, were randomized to sorafenib combined with intensive chemotherapy followed by sorafenib maintenance versus chemotherapy alone. ${ }^{59}$ The sorafenib arm had significantly longer 3-year EFS compared to placebo (40\% vs. $22 \%$, HR 0.64 , 95\% CI: $0.45-0.91, P=0.013)$, but there was no statistical difference in OS between arms.

\section{Midostaurin}

Midostaurin is a class I FLT3 inhibitor that has activity against WT and mFLT3 (ITD and TKD), KIT, PDGFR $\alpha / \beta$, VEGFR2, and members of the protein kinase C family. ${ }^{60}$ In early phase trials, midostaurin induced a reduction in peripheral and/or BM blasts (defined as a $50 \%$ reduction in either compartment) in the majority of $\mathrm{R} / \mathrm{R}$ patients with mFLT3 AML, but rarely induced deeper clinical responses. ${ }^{61,62}$ It was subsequently studied in combination with standard of care chemotherapy and in the phase III RATIFY trial, in which it improved OS without improving rates of remission or impacting rates of alloHSCT, possibly by inducing deeper remissions in those who respond. ${ }^{22,63}$ Midostaurin has changed the standard of care for newly-diagnosed mFLT3 AML, but its limited potency against the mFLT3 receptor has limited its efficacy in other circumstances. ${ }^{50}$

\section{Second generation FLT3 inhibitors}

Second generation FLT3 inhibitors immediately distinguish themselves from first generation inhibitors by virtue of their ability to more fully inhibit FLT3 in vivo. This is reflected in their capacity to trigger myeloid differentiation and their significant single-agent clinical activity.

\section{Gilteritinib}

Gilteritinib is the only FDA-approved second generation FLT3 inhibitor. It is far more potent than first generation FLT3 inhibitors, in part because it is bound to a lesser extent by plasma-protein. It has a long half-life and a large therapeutic window, achieving greater than $85 \%$ inhibition of FLT3 phosphorylation at a dose far below the maximally tolerated dose. ${ }^{64}$ While it has some activity against other tyrosine kinases including AXL, it is far more potent against mFLT3 than these other tyrosine kinases. As a result of these pharmacologic properties, gilteritinib induced higher rates of remission and better OS in the R/R setting than salvage chemotherapy, all while preserving a favorable toxicity profile. ${ }^{51}$ As a class I FLT3 inhibitor, gilteritinib is not as susceptible to the development of resistance via the acquisition of mutations in the FLT3 inhibitor, but resistance does eventually develop. Resistance most commonly develops as a result of acquired mutations in FLT3, such as the F691I/L gatekeeper mutation, or in components of the pathway downstream of the FLT3 receptor such as the RAS family. ${ }^{65}$ Resistance may also develop through the acquisition of driver mutations that activate other, unrelated pathways. Some of these may be targeted, as in the case of new IDH1/2 mutations or BCR-ABL fusions, highlighting the importance of performing mutational analyses for select, targetable genes at the time of disease progression on gilteritinib and other FLT3 inhibitors.

\section{Quizartinib}

Quizartinib is a class II, second generation FLT3 inhibitor. It is one of the most potent FLT3 inhibitors, but is ineffective against TKD mutations and is susceptible to the development of acquired resistance by the accumulation of point mutations in the $\mathrm{TKD}^{52}$ In the phase III 
QUANTUM-R study, quizartinib monotherapy was compared to salvage chemotherapy in patients with R/R FLT3mutated AML. ${ }^{66}$ Patients in the quizartinib arm had a significantly higher $(\mathrm{CRc})$ rate $(48 \%)$ than patients in the chemotherapy arm (27\%) with complete remission with incomplete hematologic recovery (CRi) predominating. Patients in the quizartinib arm were more likely to undergo alloHSCT (32\%) than those in the chemotherapy arm (11\%). The quizartinib arm also had longer OS compared to the salvage chemotherapy arm (HR 0.76, 95\% CI: $0.58-$ $0.98, P=0.02)$. The most significant adverse effects in the quizartinib arm were myelosuppression and QTc prolongation. The former probably results from quizartinib's activity against c-KIT, a receptor tyrosine kinase that is closely related to FLT3 and that is present on HSC. The QTc prolongation appears to be dose-dependent and has been a concern at the regulatory level that has delayed quizartinib's approval (although the drug is approved in Japan). Quizartinib may be particularly effective as part of an induction regimen because of its potency. The QUANTUM-first study (clinicaltrials.gov identifier: NCT03250338), a phase III study of $7+3$ and quizartinib versus $7+3$ alone, has completed enrollment and is likely to be one of the first studies of combined second generation FLT3 inhibitors and chemotherapy to yield results.

\section{Crenolanib}

Crenolanib is another potent, class I second generation FLT3 inhibitor that demonstrated clinical efficacy in a phase II trial in the R/R setting. ${ }^{67}$ In patients who had not previously received a FLT3 inhibitor, it achieved a cytogenetic CR (CRc) rate of $37 \%$ with a partial response (PR) rate of $11 \%$. Amongst patients previously treated with a FLT3 inhibitor, it achieved a CRc in $15 \%$ of patients and a $\mathrm{PR}$ in $13 \%$ of patients. These responses were probably driven by crenolanib's activity against FLT3 D835 TKD mutations, which frequently develop in patients treated with class II inhibitors. ${ }^{68}$ The patterns of resistant clones that emerged after crenolanib therapy differed from those seen in cohorts of patients treated with other TKI. ${ }^{69}$ Secondary FLT3 mutations were rare, but non-responders tended to have mutations in TET2 and IDH1/2, while patients who relapsed on crenolanib acquired mutations in these genes as well as NRAS. Crenolanib also has less activity against c-KIT and therefore is less myelosuppressive than quizartinib. In an ongoing phase II trial (clinicaltrials.gov identifier: NCT02283177) of crenolanib in combination with chemotherapy $(7+3), 85 \%$ of patients had a $\mathrm{CR}$ and 19 of 27 patients were alive and disease free at a median of 29.3 months of follow up. ${ }^{70}$

\section{FF-10101}

FF-10101 is a novel FLT3 inhibitor that covalently binds to FLT3 at the cysteine residue at $695 .{ }^{71}$ As a result, it is a potent and irreversible inhibitor of FLT3 that is unaffected by common resistance mutations, including those that occur within the TKD as well as the F691L gatekeeper mutation. It is currently being studied in a multicenter clinical trial (clinicaltrials.gov identifier: NCT03194685).

The potential for improving outcomes of acute myeloid leukemia by targeting FLT3 now and in the near future

The opportunities for more effective targeting of FLT3 will expand with the availability of new FLT3 inhibitors, but practical questions will also arise. Which inhibitor is best suited for a given clinical scenario? Is there any benefit to switching from one inhibitor to another? Should we target non-canonical FLT3 mutations or WT FLT3? Combining FLT3 inhibitors with other novel therapies also has the potential to improve efficacy while reducing toxicity. Finally, there are new immunotherapeutic approaches to targeting FLT3 on the horizon which may further impact the future of mFLT3 AML management.

\section{Selecting the most appropriate FLT3 inhibitor for the patient and the clinical scenario}

Determining which FLT3 inhibitor is most effective in the upfront setting is an area of intense interest. A FLT3 inhibitor with greater potency against FLT3 than midostaurin may suppress mFLT3 signaling more effectively, leading to better suppression or elimination of the mFLT3 clone. FL is also known to compete with FLT3 inhibitors, ${ }^{72}$ and a more potent inhibitor might compete more effectively, counteracting the protective effect of endogenous FL. Preliminary data from a phase I study of upfront gilteritinib in combination with chemotherapy demonstrate a $100 \%$ CRc in patients with de novo AML. On the other hand, midostaurin's broader anti-kinase activity may enhance its anti-proliferative effect, especially in the upfront setting when the mFLT3 clone is relatively small and the leukemia is heterogeneous. ${ }^{28}$ Trials comparing the combination of midostaurin and chemotherapy with second generation inhibitors and chemotherapy are poised to answer this question (clinicaltrials.gov identifiers: NCT04027309 and NCT02400281).

Multiple FLT3 inhibitors are also being investigated in the maintenance setting. Here too, potency is likely to be an asset, but other advantageous characteristics might include favorable toxicity profiles as well as a high threshold for the development of resistance. Class II inhibitors, for example, may be less effective in this setting due to the frequency with which mFLT3 clones acquire resistant TKD mutations.

Finally, there may be occasions for switching FLT3 inhibitors. FLT3 inhibitors have different side effect profiles that may make one more tolerable than another for a given patient. When the duration of treatment is likely to be short (as in the case of pre-transplant therapy), potency may be prioritized over tolerability and susceptibility to the development of resistance, whereas in the maintenance setting, these priorities may be inverted. Resistance patterns also differ and the emergence of a TKD mutation in response to treatment with quizartinib for example, might require a switch to a class I inhibitor. There is not yet much data on central nervous system (CNS) penetration, but the presence of CNS disease may also play a role in FLT3 inhibitor selection.

\section{Novel combinations}

FLT3 inhibitors are also being studied in combination with a number of other therapeutic partners including HMA/FLT3, Ven/FLT3, HMA/Ven/FLT3, Others + FLT3, IDH + FLT3, and other investigational agents (Table 1). HMA may be well-suited for combination therapy with FLT3 inhibitors as they have synergistic effects in vitro and appear to induce FL expression to a lesser extent than other conventional chemotherapies. Prior to the advent of second generation FLT3 inhibitors, the combination of sorafenib and azacitidine demonstrated an acceptable toxicity profile and a $46 \%$ overall response rate (ORR), lead- 
Table 1. Trials investigating the safety and efficacy of FLT3 inhibitors in combination with other therapeutic agents.

\begin{tabular}{|c|c|c|c|}
\hline Combination & Setting & Phase & Clinical Trial Number \\
\hline \multicolumn{4}{|l|}{ FLT3 Inhibitor + Chemotherapy: } \\
\hline Gilteritinib $+7+3$ vs. $7+3$ & ND AML & Phase III & NCT02236013 \\
\hline Crenolanib $+7+3$ & ND mFLT3 AML & Phase II & NCT02283177 \\
\hline Quizartinib $+7+3$ vs. $7+3$ & ND mFLT3-ITD AML & Phase III & NCT02668653 \\
\hline CPX-351 + Quizartinib & RR mFLT3-ITD AML & Phase II & NCT04209725 \\
\hline Quizartinib + High dose Ara-C and Mitoxantrone & RR mFLT3-ITD AML & Phase II & NCT03989713 \\
\hline Crenolanib + Chemotherapy vs. Chemotherapy alone & RR mFLT3 AML & Phase III & NCT03250338 \\
\hline Crenolanib + Chemotherapy vs. Midostaurin + Chemotherapy & ND mFLT3 AML & Phase III & NCT03258931 \\
\hline Cladribine, Idarubicin, Cytarabine, and Quizartinib & $\begin{array}{l}\text { ND or RR AML, acute biphenotyic } \\
\text { leukemia or high risk MDS }\end{array}$ & Phase II & NCT04047641 \\
\hline
\end{tabular}

\title{
FLT3 Inhibitor + HMA:
}

Gilteritinib + Azacitidine $v s$. Azacitidine alone

Quizartinib + Azacitidine or low-dose cytarabine

ND mFLT3 AML, ineligible for intensive induction

Phase III

NCT02752035

Phase I, IIB cohorts: RR AML, MDS, $\quad$ Phase I/II $\quad$ NCT01892371

or CMML Phase II cohort: ND or RR mFLT3 AML, MDS or CMML

\section{FLT3 Inhibitors + Novel Agents:}

Venetoclax + Quizartinib

Quizartinib + Decitabine + Venetoclax

Venetoclax + Gilteritinib

Azacitidine + Venetoclax + Gilteritinib

Gilteritinib + Atezolizumab

FLT3 Inhibitors as Maintenance:

Gilteritinib vs. Placebo

Crenolanib

Gilteritinib

$\begin{array}{r}\text { Crenolanib } \\ \hline \text { Gilteritinib }\end{array}$

RR mFLT3-ITD AML

ND or RR mFLT3 AML or MDS, ineligible for intensive induction

$$
\text { RR AML }
$$

Phase I, IIB cohorts: RR mFLT3 AML or MDS, Phase IIA cohort: ND AML

RR mFLT3 AML

mFLT3 AML post-transplant maintenance mFLT3 AML post-transplant maintenance mFLT3 AML post-CR1 maintenance

\section{FLT3 Inhibitors + Investigational Agents:}

Quizartinib + Milademetan (MDM2 inhibitor)

FLT3 inhibitor (PKC412) + MTOR inhibitor (RAD001) ND and unfit for intensive induction or RR FLT3-ITD AML
RR AML, MDS or CMML ND mFLT3 AML

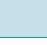

\section{Gemtuzumab + Midostaurin $+7+3$ \\ Immunotherapeutic Strategies:}

FLT3 CAR-T AMG 553

RR mFLT3 AML AML

\begin{tabular}{cc}
$\begin{array}{c}\text { Phase I/II } \\
\text { Phase I/II }\end{array}$ & NCT03735875 \\
NCT03661307 \\
Phase I & NCT03625505 \\
Phase I/II & NCT04140487 \\
\hline Phase I/II & NCT03730012
\end{tabular}

\section{Fc-optimized antibody (FLYSYN) to FLT3}

HMA: hypomethylating agent; ITD: internal tandem duplication plete remission; ND: newly diagnosed; RR: relapsed or refractory.

ing to hopes that combining HMA with newer and more potent FLT3 inhibitors may be even more effective. ${ }^{26,73}$ In two ongoing trials of quizartinib and gilteritinib in combination with azacitidine or low-dose cytarabine (clinicaltrials.gov identifiers: NCT01892371 and NCT02752035), preliminary analyses show ORR of $73 \%$ and $80 \%$, respectively. $^{74}$

There is also preclinical data to support the use of BCL2 inhibitors in combination with FLT3 inhibitors. BCL-2 inhibitors are effective in combination with HMA and low-dose cytarabine in the treatment of AML, but are ineffective as monotherapy. Resistance to BCL-2 inhibitor monotherapy is mediated by the upregulation of MCL-1. FLT3 inhibitors downregulate MCL-1 via reduced signaling through the RAS/MAPK pathway. ${ }^{75}$ Two trials of venetoclax in combination with FLT3 inhibitors (clinicaltrials.gov identifiers: NCT03625505 and NCT03735875) are currently underway. In a preliminary analysis of the for- mer, the ORR for patients with mFLT3 was $90 \%$, despite the high rate of prior exposure to FLT3 inhibitors. ${ }^{76}$ Two other trials combining venetoclax with FLT3 inhibitors and HMA are also underway (clinicaltrials.gov identifier: NCT04140487 and NCT03661307).

As the armamentarium of targeted therapies grows, it is increasingly common to find mFLT3 alongside other druggable targets. The question that arises is whether or not there is any benefit to combining FLT3 inhibitors with other targeted agents. For example, FLT3 occurs in approximately $35-40 \%$ of patients presenting with APL. ${ }^{77}$ As is the case in almost every circumstance, mFLT3 APL has a worse prognosis. Interestingly, the worse prognosis associated with mFLT3 AML is mitigated when arsenic is incorporated into the treatment regimen. ${ }^{78,79}$ This may be a quirk of arsenic and its complex mechanism of action. Another circumstance is that of concomitant FLT3 and IDH mutations. Whether the addition of a FLT3 inhibitor 
to an IDH inhibitor improves outcomes in this population is not yet known, and the question may be difficult to answer given the relative rarity of this circumstance.

\section{Targeting non-canonical FLT3 mutations and wild-type FLT3}

Trials of FLT3 inhibitors have been restricted to patients with ITD and D835 TKD mutations; however, there are a number of other less common FLT3-activating mutations. Table 2 provides an overview of non-canonical mutations of clinical significance. These mutations occur at sites within the extracellular domain, the JMD, the ATP binding pocket of the TKD1, and the activation loop of the TKD2. They are often quite close to or within sites affected by canonical mutations. For example, a series of activating point-mutations and small insertions/deletions have been identified within exon 14 of the JMD between codons Y579 and F594, the same site in which ITD are frequently inserted. Mutations adjacent to D835 have also been identified, including point mutations affecting codons R834, I836, S840, N841 and Y842. Finally, a series of mutations within the TKD 1 have been documented in the setting of acquired resistance to FLT3 inhibitors. While each of these mutations are rare, the increased use of NGS is going to increase their detection and their collective prevalence may be significant. Many of these mutations impart sensitivity or resistance to FLT3 inhibitors, emphasizing the need for better recognition and familiarity with them.

There may also be a role for FLT3 inhibitors in the treatment of WT-FLT3 AML, especially in cases where FLT3 is overexpressed. In a mouse model of myeloproliferative disease (MPD) driven by wild-type FLT3, the administration of quizartinib moderated the myeloproliferative phenotype..$^{80}$ This notion is further supported by the observation that some patients with WT-FLT3 enrolled in the phase II studies of quizartinib and gilteritinib had clinical responses. ${ }^{64,81}$ The success of this strategy is likely to depend on the potency of the FLT3 inhibitor to WT FLT3 and its capacity to compete with endogenous FLT3 ligand. A phase II study of chemotherapy plus quizartinib versus chemotherapy/placebo in patients with WT FLT3 is underway (clinicaltrials.gov identifier: NCT04107727).

\section{Tailoring therapy to minimal residual disease}

In addition to improving the rates of first remission and transplant, FLT3 inhibitors may have a role in reducing or eliminating mFLT3 minimal residual disease (MRD). The presence of MRD portends a poor prognosis in AML, and this has been demonstrated using a variety of markers and assays. ${ }^{82} \mathrm{MRD}$ in the pre-transplant setting has also been shown to correlate with higher rates of post-transplant relapse. The incorporation of MRD testing into the clinical management of AML has lagged behind that of other leukemic diseases such as chronic myleoid leukemia and acute lymphoblastic leukemia due to the heterogeneous nature of AML. MRD assays have been developed in a piecemeal fashion for specific subsets of the disease, but even these have suffered from lack of standardization across laboratories, limiting their clinical utility. Nonetheless, progress has been made in the measurement of residual mFLT3. ${ }^{83-86}$ These assays have demonstrated prognostic relevance and are poised to assume a role as road markers to guide the effective use of FLT3 inhibitors and the management of mFLT3 AML more broadly.
The potential use of FLT3-ITD as a marker of residual disease is controversial because it can be non-detectable at relapse, or, alternatively, a previously undetected ITD mutation can emerge at relapse. However, a FLT3-ITD mutation is an appealing MRD marker for several reasons: first, FLT3 is almost always the final mutation in the leukemogenic sequence and therefore is associated almost exclusively with the leukemic clone and not a preleukemic clone. Second, each individual patient's unique ITD sequence length serves as a longitudinal signature that reduces the likelihood of a false positive. Taken together, these indicate that the presence of residual FLT3-ITD of the same length as the initial FLT3-ITD mutation are indicative of residual leukemia. Third, the ability to target mFLT3 with FLT3 inhibitors and to measure the response in mFLT3 allelic burden may make quantitative FLT3 testing predictive, not just prognostic. ${ }^{63}$

With further prospective validation and standardization, FLT3-ITD MRD may be used to inform the decision to modulate our targeting of mFLT3. For example, given the higher rates of post-transplant relapse amongst patients with FLT3 MRD prior to transplant, clinicians might choose to deepen remissions with an additional cycle of treatment or by combining a FLT3 inhibitor with another agent prior to transplant. In MRD negative patients, surveillance for the re-emergence of FLT3-ITD $\mathrm{MRD}$ in the post-treatment or post-transplant setting may be used to guide the decision to re-initiate a FLT3 inhibitor to stave off clinical relapse. FLT3-ITD MRD may also serve as a valuable surrogate endpoint in trials testing new strategies to target mFLT3.

\section{Post-transplant maintenance}

The SORMAIN study is a landmark study in that it was the first to demonstrate a survival benefit to post-transplant maintenance with a FLT3 inhibitor. However, the relevance of this finding has depreciated with the rapid evolution of mFLT AML management. On one hand, the majority of patients enrolled in the study had no prior exposure to FLT3 inhibitors, and on the other, we now have far more potent FLT3 inhibitors. Therefore, the role of FLT3 inhibitors in the post-transplant setting remains uncertain and is actively being investigated. The largest of these studies is the international, phase III BMT-CTN 1506 (clinicaltrials.gov identifier: NCT02997202) study, which enrolled patients with mFLT3-ITD AML undergoing alloHSCT after first remission and randomized them to post-transplant maintenance with gilteritinib or placebo. The study has reached full accrual, and is now in follow up with preliminary results expected in 2021.

The importance of this study reflects the equipoise regarding the risks and benefits of FLT3 inhibitors in this setting. Second generation FLT3 inhibitors are well-tolerated, but long-term FLT3 inhibition may not be risk free given their role in maintenance of healthy dendritic cell. Nonetheless, there are instances in which we advocate for the off-label use of FLT3 inhibitors in the post-transplant setting. These include patients with a high risk of post-transplant relapse such as those with high FLT3ITD mutant allelic ratios and patients with FLT3-ITD MRD by flow or NGS prior to transplant. Our preference is to use gilteritinib in this setting because of its potency and tolerability compared to other FLT3 inhibitors in clinical use. 
Table 2. A list of non-canonical, activating mutations that have been found in de novo acute myeloid leukemia or in patients that progressed on treatment with a FLT3 inhibitor. The last column provides a prediction of each mutation's likely sensitivity to types of FLT3 inhibitors based upon in vitro studies.

$\begin{array}{lll}\text { Mutation } & \text { Time of Onset } & \text { Functional Impact }\end{array}$

\section{Extracellular Domain}

S451F $10 \quad$ De novo Constitutive kinase activation by unknown mechanism Relatively resistant to most FLT3 inhibitors

\section{Juxtamembrane Domain}

\begin{tabular}{|c|c|c|c|}
\hline $\mathrm{Y}_{572 \mathrm{C}^{10}}$ & De novo & Loss of JM autoinhibitory function & Sensitive to type 1 and 2 inhibitors \\
\hline $\mathrm{V} 579 \mathrm{~A} / \mathrm{G}^{10,90,91}$ & De novo & Loss of JM autoinhibitory function & Sensitive to type 1 and 2 inhibitors \\
\hline F590G, Y591D & De novo & Loss of JM autoinhibitory function & Sensitive to type 1 and 2 inhibitors \\
\hline D593N $\mathrm{N}^{91}$ & De novo & Loss of JM autoinhibitory function & Sensitive to type 1 and 2 inhibitors \\
\hline F594L $L^{90}$ & De novo & Loss of JM autoinhibitory function & Sensitive to type 1 and 2 inhibitors \\
\hline Q598_Y599del93 ${ }^{93}$ & De novo & Loss of JM autoinhibitory function & Sensitive to type 1 and 2 inhibitors \\
\hline
\end{tabular}

$\begin{array}{cc}\text { Q598_Y599del }{ }^{13} & \text { De novo } \\ \text { ATP Binding Pocket of TK Domain } 1\end{array}$

\begin{tabular}{lccc} 
F621L $^{94}$ & De novo & Unknown & Sensitive to type 1 and 2 inhibitors \\
A627P $^{94}$ & De novo & Unknown & Relatively resistant to most FLT3 inhibitors \\
\hline M664 $^{95}$ & Acquired resistance & Altered inhibitor binding & Relatively resistant to type 2 inhibitors
\end{tabular}

\begin{tabular}{lccc} 
N676 $\Delta^{9,96,97}$ & De novo, acquired resistance & $\begin{array}{c}\text { Biases activation loop toward } \\
\text { active conformation }\end{array}$ & Relatively resistant to most FLT3 inhibitors \\
\hline A680V11,98 & De novo, acquired resistance & Unknown & Unknown
\end{tabular}

F691//L $\mathrm{L}^{6599} \quad$ Acquired resistance to type 1 and 2 inhibitors $\quad$ Altered inhibitor binding Relatively resistant to most FLT3 inhibitors

$\begin{array}{lll}\text { G697R }^{97} & \text { De novo, acquired resistance } & \text { Altered inhibitor binding }\end{array}$

to type 2 inhibitors

Activation Loop of TK Domain 2

\begin{tabular}{|c|c|c|c|}
\hline $\mathrm{R} 834 \mathrm{Q}^{10}$ & De novo & Constitutive active conformation & Resistant to type 2 inhibitors, sensitive to type 1 inhibitors \\
\hline $\mathrm{D} 835 \Delta^{52,100,101}$ & $\begin{array}{l}\text { De novo, acquired resistance } \\
\text { to type } 2 \text { inhibitors }\end{array}$ & Constitutive active conformation & Resistant to type 2 inhibitors, sensitive to type 1 inhibitors \\
\hline $\mathrm{I} 836 \Delta^{91}$ & $\begin{array}{l}\text { De novo, acquired resistance } \\
\text { to type } 2 \text { inhibitors }\end{array}$ & Constitutive active conformation & Resistant to type 2 inhibitors, sensitive to type 1 inhibitors \\
\hline S840GS ${ }^{102}$ & De novo & Constitutive active conformation & Resistant to type 2 inhibitors, sensitive to type 1 inhibitors \\
\hline N841 $1^{102-105}$ & De novo & Constitutive active conformation & Resistant to type 2 inhibitors, sensitive to type 1 inhibitors \\
\hline Y $842 \Delta^{10,107}$ & $\begin{array}{c}\text { De novo, acquired resistance } \\
\text { to type } 2 \text { inhibitors }\end{array}$ & Constitutive active conformation & Resistant to type 2 inhibitors, sensitive to type 1 inhibitors \\
\hline
\end{tabular}

JM: juxtamembrane; TK: tyrosine kinase.

\section{Can allo-transplant be deferred for some?}

Our improved capacity to prognosticate and to monitor disease status, as well as the availability of potent FLT3 inhibitors, may obviate the benefits of alloHSCT in a select population of patients with mFLT3 AML. As previously discussed, there is already some compelling evidence that patients with low mFLT3-ITD allelic ratio and NPM1 mutations may not derive a survival benefit from alloHSCT. ${ }^{23,34}$ However, we believe the data are insufficient to provide a broad recommendation against alloHSCT for any particular subset of patients with mFLT3 AML. There are still a number of other variables of prognostic significance including ITD length, the presence of co-mutations, and the presence of MRD that are not consistently or reliably measured in clinical practice and which remain unaccounted for in current decision models. In the face of such uncertainty, we continue to recommend alloHSCT for the vast majority of patients with mFLT3 AML.

\section{Alternative immunotherapy approaches}

Thus far, efforts to target FLT3 have largely focused on inhibiting its signaling; however, we may also be able to take advantage of the near ubiquity of FLT3 expression (either mutant or wild type) on AML leukemic blasts and the narrow expression profile on healthy cells. Several immunotherapeutic approaches are being pursued to target the FLT3 receptor. A trial of a monoclonal FLT3 antibody is currently recruiting (clinicaltrials.gov identifier: NCT02789254). One group has developed an anti-FLT3 antibody-drug conjugate. ${ }^{87}$ FLT3-CD3 bispecific antibodies have also been developed and at least one has been demonstrated to be safe in an animal model.$^{88}$ FLT3 specific chimeric antigen receptor $\mathrm{T}$-cell (CAR-T) and T-cell modified $\mathrm{T}$-cell therapies are in development as well. In vitro data even suggest a rationale for combining these immunotherapeutic strategies with FLT3 inhibitors. mFLT3 leukemic cell lines treated with a FLT3 inhibitor increased their expression of FLT3 on their cell surfaces, which translated into better T-cell mediated cytotoxicity. ${ }^{89}$

\section{Conclusions}

FLT3 signaling plays a central role in the pathogenesis of AML. Over the last 2 decades, the presence of FLT3-activating mutations has impacted prognostication and guided management decisions. The development of more 
sophisticated diagnostic techniques has provided us with the opportunity to make more informed treatment decisions, while the advent of FLT3 inhibitors has provided us with a powerful new treatment tool. These developments also demand ever more familiarity of practising clinicians with the nuances of FLT3 biology and FLT3 inhibitor pharmacology.

\section{Disclosures}

ML: Consulting/honoraria/research funding from Astellas, Fuifilm, Daiichi-Sankyo, Amgen, Agios, Novartis. AA: No disclosures.

\section{Contributions}

$A A$ and $M L$ wrote the manuscript together.

\section{References}

1. Van Der Geer P, Hunter T, Lindberg RA. Receptor protein-tyrosine kinases and their signal transduction pathways. Ann Rev Cell Biol. 1994;10251-10337.

2. Beaudin AE, Boyer SW, Forsberg EC. Flk2/Flt3 promotes both myeloid and lymphoid development by expanding non-selfrenewing multipotent hematopoietic progenitor cells. Exp Hematol. 2014;42(3):218229.e4.

3. Papaemmanuil E, Gerstung M, Bullinger L, et al. Genomic classification and prognosis in acute myeloid leukemia. N Engl J Med. 2016;374(23):2209-2221

4. Dosil M, Wang S, Lemischka IR. Mitogenic signalling and substrate specificity of the Flk2/Flt3 receptor tyrosine kinase in fibroblasts and interleukin 3-dependent hematopoietic cells. Mol Cell Biol. 1993;13(10):6572-6585

5. Rosnet O, Bühring HJ, De Lapeyriére $O$, et al. Expression and signal transduction of the FLT3 tyrosine kinase receptor. Acta Haematol. 1996;95(3-4):218-223.

6. Tsapogas P, Mooney CJ, Brown G, Rolink A. The cytokine Flt3-ligand in normal and malignant hematopoiesis. Int J Mol Sci. 2017;18(6):1115.

7. Thiede C, Steudel C, Mohr B, et al. Analysis of FLT3-activating mutations in 979 patients with acute myelogenous leukemia: Association with FAB subtypes and identification of subgroups with poor prognosis. Blood. 2002;99(12):4326-4335.

8. Schnittger S, Schoch C, Dugas M, et al. Analysis of FLT3 length mutations in 1003 patients with acute myeloid leukemia: correlation to cytogenetics, FAB subtype, and prognosis in the AMLCG study and usefulness as a marker for the detection of minimal residual disease. Blood. 2002;100(1):5966.

9. Heidel F, Solem FK, Breitenbuecher F, et al. Clinical resistance to the kinase inhibitor PKC412 in acute myeloid leukemia by mutation of Asn-676 in the FLT3 tyrosine kinase domain. Blood. 2006;107(1):293-300.

10. Fröhling S, Scholl C, Levine RL, et al. Identification of driver and passenger mutations of FLT3 by high-throughput DNA sequence analysis and functional assessment of candidate alleles. Cancer Cell. 2007;12(6): 501-513.

11. Fiskus W, Sharma S, Qi J, et al. BET protein antagonist JQ1 is synergistically lethal with FLT3 tyrosine kinase inhibitor (TKI) and overcomes resistance to FLT3-TKI in AML cells expressing FLT-ITD. Mol Cancer Ther. 2014;13(10):2315-2327.

12. Carow CE, Levenstein M, Kaufmann SH, et al. Expression of the hematopoietic growth factor receptor FLT3 (STK-1/Flk2) in human leukemias. Blood 1996;87(3):1089-1096.

13. Gilliland DG, Griffin JD. The roles of FLT3 in hematopoiesis and leukemia. Blood. 2002;100(5):1532-1542.

14. Kuchenbauer F, Kern W, Schoch C, et al.
Detailed analysis of FLT3 expression levels in acute myeloid leukemia. Haematologica. 2005;90(12):1617-1625.

15. Tarlock K, Alonzo TA, Loken MR, et al. Disease characteristics and prognostic implications of cell-surface FLT3 receptor (CD135) expression in pediatric acute myeloid leukemia: a report from the Children's Oncology Group. Clin Cancer Res. 2017;23(14):3649-3656.

16. Cheng J, Qu L, Wang J, Cheng L, Wang Y. High expression of FLT3 is a risk factor in leukemia. Mol Med Rep. 2018;17(2):28852892.

17. Kandeel EZ, El Sayed G, Elsharkawy N, et al Impact of FLT3 receptor (CD135) Detection by Flow Cytometry on Clinical Outcome of Adult Acute Myeloid leukemia patients. Clin Lymphoma Myeloma Leuk. 2018;18(8):541-547.

18. Daver N, Strati P, Jabbour E, et al. FLT3 mutations in myelodysplastic syndrome and chronic myelomonocytic leukemia. Am Hematol. 2013;88(1):56-59.

19. Rosenzwajg M, Camus S, Guigon M, Gluckman JC. The influence of interleukin (IL)-4, IL-13, and Flt3 ligand on human dendritic cell differentiation from cord blood CD34+ progenitor cells. Exp Hematol. 1998;26(1):63-72

20. McKenna HJ, Stocking KL, Miller RE, et al. Mice lacking flt3 ligand have deficient hematopoiesis affecting hematopoietic progenitor cells, dendritic cells, and natural killer cells. Blood. 2000;95(11):3489-3497.

21. Levis M. FLT3/ITD AML and the law of unintended consequences. Blood. 2011;117(26):6987-6990.

22. Stone RM, Mandrekar SJ, Sanford BL, et al. Midostaurin plus chemotherapy for acute myeloid leukemia with a FLT3 mutation. N Engl J Med. 2017;377(5):454-464

23. Döhner K, Thiede C, Jahn N, et al. Impact of NPM1/FLT3-ITD genotypes defined by the 2017 European LeukemiaNet in patients with acute myeloid leukemia. Blood. 2020;135(5):371-380.

24. Chyla BJ, Harb J, Mantis C, et al. Response to venetoclax in combination with low intensity therapy (LDAC or HMA) in untreated patients with acute myeloid leukemia patients with IDH, FLT3 and other mutations and correlations with BCL2 family expression. Blood. 2019;134 (Supplement_1):546-546.

25. DiNardo CD, Tiong IS, Quaglieri A, et al. Molecular patterns of response and treatment failure after frontline venetoclax combinations in older patients with AML. Blood. 2020;135(11):791-803.

26. Ravandi F, Alattar ML, Grunwald MR, et al. Phase 2 study of azacytidine plus sorafenib in patients with acute myeloid leukemia and FLT-3 internal tandem duplication mutation. Blood. 2013;121(23):4655-4662

27. National Comprehensive Cancer Network. Acute Myeloid Leukemia (Version 3.2020 December 23, 2019). Available from: https://www.nccn.org/professionals/physician_gls/pdf/aml.pdf (Last accessed June 16,
2020).

28. Pratz KW, Sato T, Murphy KM, Stine A, Rajkhowa T, Levis M. FLT3-mutant allelic burden and clinical status are predictive of response to FLT3 inhibitors in AML. Blood. 2010;115(7):1425-1432.

29. Ding L, Ley TJ, Larson DE, et al. Clonal evolution in relapsed acute myeloid leukaemia revealed by whole-genome sequencing. Nature. 2012;481(7382):506-510.

30. Döhner H, Estey E, Grimwade D, et al. Diagnosis and management of AML in adults: 2017 ELN recommendations from an international expert panel. Blood. 2017;129 (4):424-447.

31. Meshinchi S, Arceci RJ, Sanders JE, et al. Role of allogeneic stem cell transplantation in FLT3/ITD-positive AML. Blood. 2006;108(1):400.

32. DeZern AE, Sung A, Kim S, et al. Role of allogeneic transplantation for FLT3/ITD acute myeloid leukemia: Outcomes from 133 consecutive newly diagnosed patients from a single institution. Biol Blood Marrow Transplant. 2011;17(9):1404-1409.

33. Koreth J, Schlenk R, Kopecky KJ, et al. Allogeneic stem cell transplantation for acute myeloid leukemia in first complete remission: systematic review and metaanalysis of prospective clinical trials. JAMA. 2009;301(22):2349-2361.

34. Schlenk RF, Kayser S, Bullinger L, et al. Differential impact of allelic ratio and insertion site in FLT3-ITD-positive AML with respect to allogeneic transplantation. Blood. 2014;124(23):3441-3449.

35. Oran B, Cortes J, Beitinjaneh A, et al. Allogeneic transplantation in first remission improves outcomes irrespective of FLT3ITD allelic ratio in FLT3-ITD-positive acute myelogenous leukemia. Biol Blood Marrow Transplant. 2016;22(7):1218-1226

36. Gaballa S, Saliba R, Oran B, et al. Relapse risk and survival in patients with FLT3 mutated acute myeloid leukemia undergoing stem cell transplantation. Am J Hematol. 2017:92(4):331-337.

37. Kayser S, Schlenk RF, Londono MC, et al. Insertion of FLT3 internal tandem duplication in the tyrosine kinase domain-1 is associated with resistance to chemotherapy and inferior outcome. Blood. 2009;114(12):23862392.

38. Breitenbuecher F, Schnittger S, Grundler R, et al. Identification of a novel type of ITD mutations located in nonjuxtamembrane domains of the FLT3 tyrosine kinase receptor. Blood. 2009;113(17):4074-4077.

39. Griffith J, Black J, Faerman C, et al. The structural basis for autoinhibition of FLT3 by the juxtamembrane domain. Mol Cell. 2004;13(2):169-178.

40. Stirewalt DL, Kopecky KJ, Meshinchi S, et al. Size of FLT3 internal tandem duplication has prognostic significance in patients with acute myeloid leukemia. Blood. 2006;107 (9):3724-3726.

41. Chen F, Sun J, Yin C, et al. Impact of FLT3ITD allele ratio and ITD length on therapeutic outcome in cytogenetically normal AML 
patients without NPM1 mutation. Bone Marrow Transplant. 2020;55(4):740-748.

42. Schwartz GW, Manning B, Zhou Y, et al. Classes of ITD predict outcomes in AML patients treated with FLT3 inhibitors. Clin Cancer Res. 2019;25(2):572-583.

43. Liu SB, Dong HJ, Bao XB, et al. Impact of FLT3-ITD length on prognosis of acute myeloid leukemia. Haematologica. 2019;104(1):e9-e12

44. Murphy KM, Levis M, Hafez MJ, et al. Detection of FLT3 internal tandem duplication and D835 mutations by a multiplex polymerase chain reaction and capillary electrophoresis assay. J Mol Diagnostics. 2003:5(2):96-102.

45. Polz MF, Cavanaugh CM. Bias in templateto-product ratios in multitemplate PCR. Appl Environ Microbiol. 1998;64(10)37243730 .

46. Genomic and epigenomic landscapes of adult de novo acute myeloid leukemia. N Engl J Med. 2013;368(22):2059-2074.

47. Guryanova OA, Shank K, Spitzer B, et al. DNMT3A mutations promote anthracycline resistance in acute myeloid leukemia via impaired nucleosome remodeling. Nat Med. 2016;22(12):1488-1495

48. Garg S, Reyes-Palomares A, He L, et al. Hepatic leukemia factor is a novel leukemic stem cell regulator in DNMT3A, NPM1, and FLT3-ITD triple-mutated AML. Blood. 2019;134(3):263-276.

49. Burchert A, Bug G, Finke J, et al. Sorafenib as maintenance therapy post allogeneic stem cell transplantation for FLT3-ITD positive AML: results from the randomized, doubleblind, placebo-controlled multicentre Sormain trial. Blood. 2018;132(Supplement 1):661.

50. Maziarz RT, Fernandez H, Patnaik MM, et al. Radius: midostaurin (mido) plus standard of care (SOC) after allogeneic stem cell transplant (alloSCT) in patients (pts) with FLT3internal tandem duplication (ITD)-mutated acute myeloid leukemia (AML). Biol Blood Marrow Transplant. 2019;25(3):S11-S12.

51. Perl AE, Martinelli G, Cortes JE, et al. Gilteritinib or chemotherapy for relapsed or refractory FLT3 -mutated AML. N Engl J Med. 2019;381(18):1728-1740.

52. Smith CC, Paguirigan A, Jeschke GR, et al. Heterogeneous resistance to quizartinib in acute myeloid leukemia revealed by singlecell analysis. Blood. 2017;130(1):48-58.

53. Levis M, Brown P, Smith BD, et al. Plasma inhibitory activity (PIA): a pharmacodynamic assay reveals insights into the basis for cytotoxic response to FLT3 inhibitors. Blood. 2006;108(10):3477-3483.

54. Pratz KW, Cortes J, Roboz GJ, et al. A pharmacodynamic study of the FLT3 inhibitor KW-2449 yields insight into the basis for clinical response. Blood. 2009;113(17):39383946.

55. Lee LY, Hernandez D, Rajkhowa T, et al. Preclinical studies of gilteritinib, a next-generation FLT3 inhibitor. Blood. 2017;129(2):257-260.

56. Levis M. Quizartinib for the treatment of FLT3/ITD acute myeloid leukemia. Futur Oncol. 2014;10(9):1571-1579.

57. O'Farrell AM, Foran JM, Fiedler W, et al. An innovative phase I clinical study demonstrates inhibition of FLT3 phosphorylation by SU11248 in acute myeloid leukemia patients. Clin Cancer Res. 2003;9(15):54655476.

58. Smith BD, Levis M, Beran M, et al. Singleagent CEP-701, a novel FLT3 inhibitor, shows biologic and clinical activity in patients with relapsed or refractory acute myeloid leukemia. Blood. 2004;103(10): 3669-3676.

59. Röllig C, Serve H, Hüttmann A, et al Addition of sorafenib versus placebo to standard therapy in patients aged 60 years or younger with newly diagnosed acute myeloid leukaemia (SORAML): a multicentre, phase 2, randomised controlled trial Lancet Oncol. 2015;16(16):1691-1699.

60. Rydapt [package insert]. East Hanover, NJ Novartis Pharmaceuticals Corporation. 2017. https://www.accessdata.fda.gov/ drugsatfda_docs/label/2017/207997s000lbl. pdf.

61. Stone RM, DeAngelo DJ, Klimek V, et al Patients with acute myeloid leukemia and an activating mutation in FLT3 respond to a small-molecule FLT3 tyrosine kinase inhibitor, PKC412. Blood. 2005;105(1):54-60.

62. Fischer T, Stone RM, DeAngelo DJ, et al. Phase IIB trial of oral midostaurin (PKC412), the FMS-like tyrosine kinase 3 receptor (FLT3) and multi-targeted kinase inhibitor, in patients with acute myeloid leukemia and high-risk myelodysplastic syndrome with either wild-type or mutated FLT3. J Clin Oncol. 2010;28(28):4339-4345.

63. Levis M, Shi W, Chang K, et al. FLT3 inhibitors added to induction therapy induce deeper remissions. Blood. 2020;135 (1):75-78

64. Perl AE, Altman JK, Cortes J, et al. Selective inhibition of FLT3 by gilteritinib in relapsed or refractory acute myeloid leukaemia: a multicentre, first-in-human, open-label, phase 1-2 study. Lancet Oncol. 2017;18(8): 1061-1075

65. MacMahon CM, Canaani J, Rea B, et al Mechanisms of acquired resistance to gilteritinib therapy in relapsed and refractory FLT3-mutated acute myeloid leukemia. Blood. 2017;130(Supplement 1):295-295.

66. Cortes JE, Khaled S, Martinelli G, et al. Quizartinib versus salvage chemotherapy in relapsed or refractory FLT3-ITD acute myeloid leukaemia (QuANTUM-R): a multicentre, randomised, controlled, open-label, phase 3 trial. Lancet Oncol. 2019;20(7):984997.

67. Cortes JE, Kantarjian HM, Kadia TM, et al. Crenolanib besylate, a type I pan-FLT3 inhibitor, to demonstrate clinical activity in multiply relapsed FLT3-ITD and D835 AML. I Clin Oncol. 2016:34(15 suppl):7008.

68. Galanis A, Ma H, Rajkhowa $\mathrm{T}$, et al. Crenolanib is a potent inhibitor of flt 3 with activity against resistance-Conferring point mutants. Blood. 2014;123(1):94-100

69. Zhang H, Savage S, Schultz AR, et al Clinical resistance to crenolanib in acute myeloid leukemia due to diverse molecular mechanisms. Nat Commun. 2019;10(1):244

70. Goldberg AD, Coombs CC, Wang ES, et al. Younger patients with newly diagnosed FLT3-mutant AML treated with crenolanib plus chemotherapy achieve adequate free crenolanib levels and durable remissions. Blood. 2019;134(Supplement_1):1326

71. Yamaura T, Nakatani T, Uda K, et al. A novel irreversible FLT3 inhibitor, FF-10101, shows excellent efficacy against AML cells with FLT3 mutations. Blood. 2018;131(4):426438.

72. Sato T, Yang X, Knapper S, et al. FLT3 ligand impedes the efficacy of FLT3 inhibitors in vitro and in vivo. Blood. 2011;117(12):32863293.

73. Ueno Y, Mori M, Kamiyama Y, Kaneko N, Isshiki E, Takeuchi M. Gilteritinib (ASP2215), a Novel FLT3/AXL Inhibitor:
Preclinical Evaluation in Combination with Azacitidine in Acute Myeloid Leukemia. Blood. 2016;128(22):2830-2830.

74. Swaminathan M, Kantariian HM, Daver N, et al. The combination of quizartinib with azacitidine or low-dose cytarabine is highly active in patients (Pts) with FLT3-ITD mutated myeloid leukemias: interim report of a phase I/II trial. Blood. 2017;130 (Supplement 1):723.

75. Ma J, Zhao S, Qiao X, et al. Inhibition of Bcl2 synergistically enhances the antileukemic activity of midostaurin and gilteritinib in preclinical models of FLT3-mutated acute myeloid leukemia. Clin Cancer Res. 2019:25(22):6815-6826.

76. Perl AE, Daver NG, Pratz KW, et al Venetoclax in combination with gilteritinib in patients with relapsed/refractory acute myeloid leukemia: a phase $1 \mathrm{~b}$ study. Blood. 2019;134(Supplement_1):3910.

77. Breccia M, Loglisci G, Loglisci MG, et al. FLT3-ITD confers poor prognosis in patients with acute promyelocytic leukemia treated with AIDA protocols: long-term follow-up analysis. Haematologica. 2013;98(12):e161.

78. Daver N, Kantarjian H, Marcucci G, et al Clinical characteristics and outcomes in patients with acute promyelocytic leukaemia and hyperleucocytosis. Br Haematol. 2015;168(5):646-653.

79. Esnault C, Rahmé R, Rice KL, et al. FLT3ITD impedes retinoic acid, but not arsenic responses in murine acute promyelocytic leukemias. Blood. 2019;133(13):1495-1506.

80. Taylor SJ, Dagger SA, Thien CBF, Wikstrom ME, Langdon WY. Flt3 inhibitor AC220 is a potent therapy in a mouse model of myeloproliferative disease driven by enhanced wild-type Flt3 signaling. Blood. 2012;120 (19):4049-4057.

81. Cortes J, Perl AE, Döhner $\mathrm{H}$, et al Quizartinib, an FLT3 inhibitor, as monotherapy in patients with relapsed or refractory acute myeloid leukaemia: an open-label, multicentre, single-arm, phase 2 trial. Lancet Oncol. 2018;19(7):889-903.

82. Schuurhuis GJ, Heuser M, Freeman S, et al Minimal/measurable residual disease in AML: a consensus document from the European LeukemiaNet MRD Working Party. Blood. 2018;131(12):1275-1291.

83. Bibault JE, Figeac M, Hélevaut N, et al. Nextgeneration sequencing of FLT3 internal tandem duplications for minimal residual disease monitoring in acute myeloid leukemia. Oncotarget. 2015;6(26):22812-22821.

84. Thol F, Gabdoulline R, Liebich A, et al. Measurable residual disease monitoring by NGS before allogeneic hematopoietic cell transplantation in AML. Blood. 2018;132 (16):1703-1713

85. Levis MJ, Perl AE, Altman JK, et al. A nextgeneration sequencing-based assay for minimal residual disease assessment in AML patients with FLT3 -ITD mutations. Blood Adv. 2018;2(8):825-831.

86. Blätte TJ, Schmalbrock LK, Skambraks S, et al. getITD for FLT3-ITD-based MRD monitoring in AML. Leukemia. 2019;33(10):25352539

87. Rudra-Ganguly N, Lowe C, Virata C, et al. AGS62P1, a novel anti-FLT3 antibody drug conjugate, employing site specific conjugation, demonstrates preclinical antitumor efficacy in AML tumor and patient derived xenografts. Blood. 2015;126(23): 3806-3806.

88. Yeung YA, Krishnamoorthy V, Dettling D, et al. An pptimized full-length FLT3/CD3 bispecific antibody demonstrates potent anti- 
